\title{
In vitro and in vivo growth inhibition of prostate cancer by the small molecule imiquimod
}

\author{
JU-HEE HAN $^{1}$, JUNGLIM LEE ${ }^{2}$, SOO-JIN JEON ${ }^{2}$, EUN-SUN CHOI $^{3}$, SUNG-DAE CHO $^{3}$, \\ BO-YEON KIM ${ }^{4}$, DONG-JAE KIM ${ }^{4,5}$, JAE-HAK PARK ${ }^{1}$ and JONG-HWAN PARK ${ }^{4,5}$
}

\author{
${ }^{1}$ Department of Laboratory Animal Medicine, College of Veterinary Medicine, Seoul National University, Seoul 151-742; \\ ${ }^{2}$ Department of Microbiology, College of Medicine, Konyang University, Daejeon 302-711; ${ }^{3}$ Department of Oral Pathology, \\ School of Dentistry and Institute of Oral Bioscience, Chonbuk National University, Jeon-ju 561-756; \\ ${ }^{4}$ World Class Institute, Korea Research Institute of Bioscience and Biotechnology, Ochang-Eup, \\ Cheongwon-Gun, Choongbuk 363-883; ${ }^{5}$ Department of Biochemistry, College of Medicine, \\ Konyang University, Daejeon 302-711, Republic of Korea
}

Received February 1, 2013; Accepted March 21, 2013

DOI: $10.3892 /$ ijo.2013.1898

\begin{abstract}
Prostate cancer is the second leading cause of cancer death in men worldwide. In the present study, we examined in vitro and in vivo antitumor effect of the small molecule imiquimod, also known as a TLR7 agonist, against prostate cancer. Imiquimod inhibited the growth of mouse (TRAMP-C2) and human (PC-3) prostate cancer cells. Treatment with imiquimod induced cell cycle arrest at the $\mathrm{G}_{2} / \mathrm{M}$ phase in TRMPA-C2 cells, confirmed by the changes of $\mathrm{G}_{2} / \mathrm{M}$ checkpoint regulators such as reduction of cyclin B1 expression and increase of phospho-CDC2 and p21 in TRAMP-C2 cells treated with imiquimod. Flow cytometry and western blot analysis revealed that imiquimod induced direct apoptosis in TRAMP-C2 cells via a mitochondrial-dependent pathway. Intratumoral injection with imiquimod reduced significantly tumor growth and increased apoptotic cells in mice subcutaneously implanted with TRAMP-C2 cells. Our results indicate that imiquimod can be an alternative therapeutic for locally generated prostate cancer.
\end{abstract}

\section{Introduction}

Prostate cancer is the most commonly diagnosed male malignancy and the second cause of malignant male death

Correspondence to: Professor Jong-Hwan Park, Department of Biochemistry, College of Medicine, Konyang University, 28, Wonangmaeul-1 Road, Seo-gu, Daejeon 302-711, Republic of Korea E-mail: jonpark@konyang.ac.kr

Professor Jae-Hak Park, Department of Laboratory Animal Medicine, College of Veterinary Medicine and KRF Zoonotic Disease Priority Research Institute, Seoul National University, Gwanak-ro 1, Gwanak-gu, Seoul 151-742, Republic of Korea

E-mail:pjhak@snu.ac.kr

Key words: imiquimod, prostate cancer, apoptosis, cell cycle arrest worldwide after lung cancer (1). Infectious agents, physical trauma, hormones and a break of immune tolerance to prostate antigen are considered as the cause of prostate cancer (2). There are several therapeutic options such as radical prostatectomy, radiation and hormonal therapy for prostate cancer (3). However, these protocols have a limitation for metastatic and hormone refractory prostate cancer. Hormonal therapy in the form of medical or surgical castration can induce significant long-term remissions, but androgen-independent patients ultimately develop metastatic prostate cancer resulting in death due to widespread metastases (3-5). Chemotherapy is also effective but long-term use is not feasible due to its toxicity (6). Therefore, the development of alternative therapeutics are required for prostate cancer.

Imiquimod is a low-molecular-weight compound belonging to the imidazoquinolines family. It was first identified as a compound that has anti-viral activity in guinea pigs infected with herpes simplex virus, and has been successfully used for the treatment of genital warts caused by human papilloma virus in the clinic $(7,8)$. In addition, recent studies have attracted considerable interest owing to their profound antitumoral activities (9-11). Imiquimod exerts antitumor effect by activating immune response to suppress tumor growth in a variety of transplantable tumors (9), it also has direct proapoptotic activity against various tumor cell populations in vitro and in vivo $(12,13)$. The $5 \%$ imiquimod cream that is commercially available has been successfully used for the treatment of several cancers including basal cell carcinomas and melanoma (11,14-16).

Antitumor effects of the imidazoquinolines family have been demonstrated in urogenital cancers including bladder cancer and renal cell carcinoma (17-21). Treatment with an imidazoquinoline (3M-011) downregulated c-Myc expression in bladder cancer cells and reduced its transcriptional activity (21). It also significantly suppressed in vivo tumor growth in a mouse model for orthotopic bladder cancer (21). Imiquimod also induced apoptosis and cytokines production 
in various bladder cancer cell lines and effectively inhibited in vivo tumor growth (17). An imidazoquinoline also enhanced in vivo apoptosis and increased lymphocytic infiltration and proinflammatory cytokine production in a mouse model of renal cell carcinoma (22). However, the effect of imidazoquinolines on the growth of prostate cancer has not been studied. Therefore, in this study, we evaluated in vitro and in vivo antitumor effect of an imidazoquinoline, imiquimod, against prostate cancer.

\section{Materials and methods}

Mice. Specific pathogen-free (SPF) C57BL/6 mice were purchased from Koatech (Pyeongtaek, Korea). All animal studies were approved, and followed the regulations of the Institutional Animal Care and Use Committee in Konyang University.

Cell lines and reagents. Transgenic adenocarcinoma of the mouse prostate (TRAMP) model-derived prostatic epithelial cell line (TRAMP-C2) (23) and the metastatic human prostate cancer cell line (PC3) were purchased from the American Type Culture Collection (Manassas, VA, USA). TRAMP-C2 cells were cultured in Dulbecco's modified Eagle's medium (DMEM, Gibco, San Diego, CA, USA) with $10 \%$ fetal bovine serum, $1 \%$ penicillin-streptomycin, $5 \%$ Nu-serum IV (Collaborative Biomedical Products, Bedford, MA, USA), $5 \mu \mathrm{g} / \mathrm{ml}$ insulin (Sigma-Aldrich Co., St. Louis, MO) and $10 \mathrm{nM}$ dihydrotestosterone (Sigma-Aldrich Co.) (24). PC3 cells were maintained in RPMI-1640 medium supplemented with $10 \%$ fetal bovine serum (Gibco) and $1 \%$ penicillin-streptomycin. Imiquimod was purchased from InvivoGen (San Diego, CA, USA) and dissolved in ultrapure water as a stock solution at a concentration of $5 \mathrm{mg} / \mathrm{ml}$.

MTT assay. The effects of imiquimod (1-10 $\mu \mathrm{g} / \mathrm{ml})$ on TRAMP-C2 cells growth were determined by MTT (3-[4,5-dimethylthiazol-2-yl]-2,5-diphenyltetrazolium bromide, Amresco $^{\circledast}$, Solon, OH, USA) assay. TRAMP-C2 cells were plated into 48-well tissue culture plates at concentration of $5 \times 10^{4}$ cells/well. After $12 \mathrm{~h}$, TRAMP-C2 cells were treated with various concentration of imiquimod for 24,48 and $72 \mathrm{~h}$. The cell culture supernatant was removed and cells were incubated with $5 \mathrm{mg} / \mathrm{ml}$ MTT solution for $4 \mathrm{~h}$. At the end of incubation, MTT solution was aspirated and then cells were mixed with dimethyl sulfoxide (DMSO, Amresco). The dye absorption was quantified using an automatic microplate spectrophotometer (Berthold Technologies $\mathrm{GmbH}$, Vienna, Austria) at $540 \mathrm{~nm}$. For TLR7 inhibitor treatment, TRAMP-C2 cells were pretreated with various concentration chloroquine for $2 \mathrm{~h}$ and then imiquimod $(10 \mu \mathrm{g} / \mathrm{ml})$ was added to the medium with chloroquine for $72 \mathrm{~h}$. Cell growth was determined by MTT assay as described above.

Flow cytometry. Imiquimod-treated TR AMP-C 2 cells were stained with PI (Sigma-Aldrich Co.) or PI/Annexin V-FITC (BD Bioscience, Franklin Lakes, NJ) and analyzed by flow cytometry (BD LSR Flow cytometer, San Jose, CA, USA).
Western blot analysis. TRAMP-C2 cells were plated into $60-\mathrm{mm}$ culture dish about $2 \times 10^{5}$ cells/well. After overnight incubation, the culture medium was replaced with fresh media and cells were treated with imiquimod $(20 \mu \mathrm{g} / \mathrm{ml})$. The cells were lysed $0,12,24$ and $48 \mathrm{~h}$ after stimulation using lysis buffer with $1 \%$ Nonidet P-40, complete protease inhibitor cocktail (Roche, Basel, Switzerland) and $2 \mathrm{mM}$ dithiothreitol. Cell lysates were incubated on ice for $30 \mathrm{~min}$ and centrifuged for $15 \mathrm{~min}$ at $13,000 \mathrm{rpm}$. The protein-containing supernatant was harvested and the total protein amount was quantified using Bradford assay kit (Bio-Rad, Hercules, CA, USA). Cell lysates were added with sample buffer and loaded onto a 10 or $12 \%$ SDS-PAGE gel. After electrophoresis, proteins were transferred onto polyvinylidene fluoride (PVDF) membrane and detected with the following antibodies: cleaved-PARP, caspase-3, caspase-7, caspase-9, cyclinB1 and phospo-CDC2 (Abcam, Cambridge, UK), p21 (Cell Signaling, Beverly, MA, USA), and anti- $\beta$-actin (Santa Cruz Biotechnology, Santa Cruz, CA, USA) and goat anti-rabbit IgG-HRP (Santa Cruz Biotechnology). The blots were developed using ECL substrate (Thermo Scientific, Waltham, MA, USA).

Cytokine production. The culture supernatants of imiquimod-treated TRAMP-C2 cells with or without cloroquine pretreatment at the concentration of 1,10 and $50 \mathrm{nM}$ were obtained and kept at $-20^{\circ} \mathrm{C}$ until cytokine measurement. The concentration of IL- 6 in culture supernatants was determined using a commercial DuoSet ELISA Development kits (R\&D Systems, Minneapolis, MN, USA) according to the manufacturer's instructions.

In vivo antitumor efficacy. C57BL/6 mice received subcutaneous (s.c.) single injection of TRAMP-C2 cells ( $1 \times 10^{6}$ cells/mouse in $100 \mu 1$ of injectable saline) into the shaven right flank and tumor growth was monitored. On day 10 after tumor implantation, imiquimod was injected intratumorally at $50 \mu \mathrm{g}$ daily for 9 days. Tumors were injected in a different site for each treatment day. Tumor length (L) and width (W) were measured and tumor weight (WR) was calculated twice a week as follows: $\mathrm{WR}=1 / 2 \times \mathrm{L} \times \mathrm{W} 2$.

Histological analysis. Tumor mass were removed, fixed in $10 \%$ formalin for $24 \mathrm{~h}$, and processed in a standard alcohol-xylene series. The tissues were then embedded in paraffin, and 3- $\mu \mathrm{m}$ sections were prepared, each of which was stained with H\&E (Sigma-Aldrich Co.).

TUNEL assay. Sections were stained for apoptotic cells by a modified terminal deoxynucleotidyl transferase-mediated dUTP-biotin nick end-labeling (TUNEL) assay using the ApopTag Peroxidase In Situ Apoptosis Detection Kit (Millipore, Billerica, MA, USA) following the manufacturer's instructions. Then sections were counterstained with Methyl Green (Sigma-Aldrich Co.).

Statistical analysis. All assays were derived from at least three independent experiments. Statistical comparisons among the different values were perform using the Prism 5 GraphPad software (San Diego, CA, USA). Data are presented as mean $\pm \mathrm{SD}$. 
A

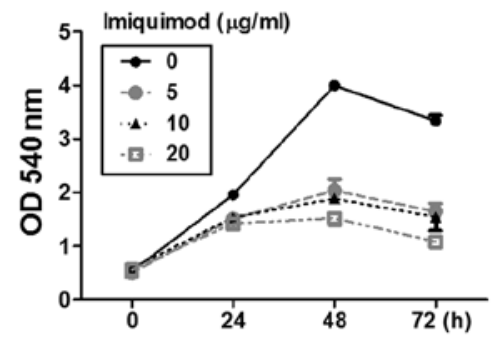

B

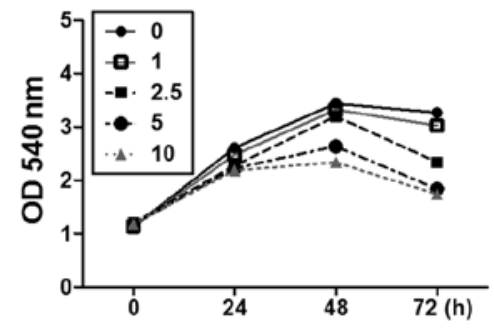

C

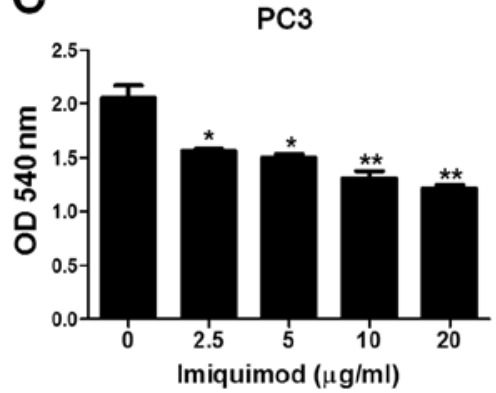

Figure 1. Imiquimod inhibits cell proliferation in prostate cancer cell lines. (A and B) TRAMP-C2 or (C) PC3 cells were treated with the indicated concentration of imiquimod for 24,48 and $72 \mathrm{~h}$. Cell growth inhibition was assessed by MTT assay at (A) high dose and (B) low dose of imiquimod treatment. The results presented here are from one representative experiment of three independent experiments and are presented as mean $\pm \mathrm{SD}$. ${ }^{*} \mathrm{P}<0.05,{ }^{* *} \mathrm{P}<0.01$.

\section{Results}

Imiquimod inhibits the growth of TRAMP-C2 cells. Inhibitory effect of imiquimod on the growth of TRAMP-C2 cells was examined by MTT assay. The growth curve of untreated cells increased rapidly by $48 \mathrm{~h}$ and slightly decreased at $72 \mathrm{~h}$. Treatment at doses over $5 \mu \mathrm{g} / \mathrm{ml}$ of imiquimod delayed the cell growth by $48 \mathrm{~h}$ (Fig. 1A). To determine more definite dose-dependent effect of imiquimod on the cell growth, the experiment was repeated with a narrow dose range of imiquimod, 1 and $2.5 \mu \mathrm{g} / \mathrm{ml}$ of imiquimod did not affect the cell growth by $48 \mathrm{~h}$ post-treatment (Fig. 1B). However, the cell growth was more reduced by a treatment with $2.5 \mu \mathrm{g} / \mathrm{ml}$ of imiquimod at $72 \mathrm{~h}$ post-treatment, as compared with that in untreated cells (Fig. 1B). The growth of PC 3 cells, a human prostate cancer cell line, was inhibited by imiquimod at $72 \mathrm{~h}$ post-treatment in a dose-dependent manner (Fig. 1C).

Imiquimod leads to $G_{2} / M$ cell cycle arrest in TRAMP-C2 cells. We next investigated the effect of imiquimod on the cell cycle arrest in TRAMP-C2 cells. The cells were treated with various doses of imiquimod for $48 \mathrm{~h}$ and DNA contents for cell cycle arrest were determined by FACS analysis. The cell percentage in $\mathrm{G}_{1}$ phase was reduced in imiquimodtreated TRAMP-C2 cells (Fig. 2A and B). In contrast, imiquimod increased the cell percentage in $\mathrm{G}_{2} / \mathrm{M}$ phase dose-dependently, as compared with untreated cells (Fig. 2A and $\mathrm{B})$. In addition, western blot analysis was performed to determine the changes of expression or activation of $G_{2} / M$ cell cycle-specific markers. Results showed that the expression of cyclin B1, which promotes nuclear accumulation and initiation of mitosis (25), was decreased in TRAMP-C2 cells by imiquimod at $48 \mathrm{~h}$ post-treatment (Fig. 2C). Moreover, imiquimod enhanced the phosphorylation of $\mathrm{CDC} 2$, which is a master regulatory kinase on the control of the $\mathrm{G}_{2} / \mathrm{M}$ transition (26), from $6 \mathrm{~h}$ after treatment (Fig. 2C). The expression of cell cycle regulator p21 was also increased by imiquimod from $12 \mathrm{~h}$ after treatment (Fig. 2C). These findings suggest that imiquimod may suppress the growth of prostate cancer cells via $\mathrm{G}_{2} / \mathrm{M}$ cell cycle arrest.

Imiquimod induces mitochondria-dependent apoptosis in prostate cancer cells. Previous studies showed that
A
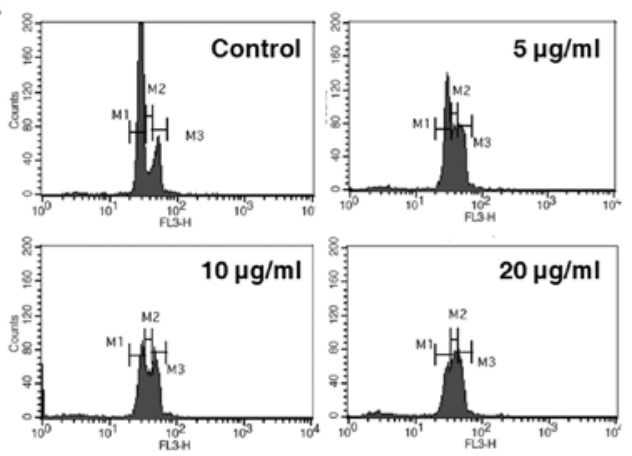

B

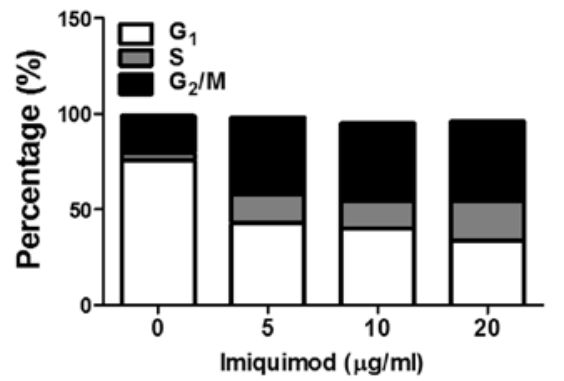

C Imiquimod

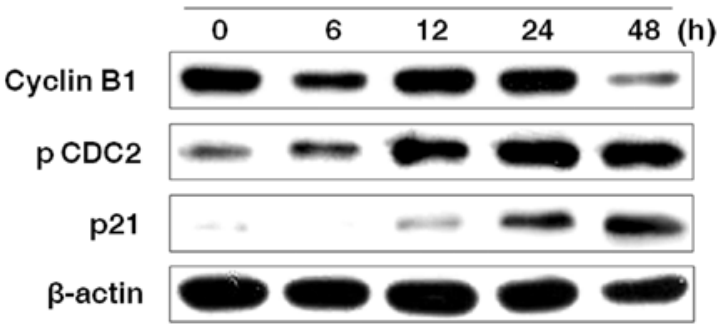

Figure 2. Imiquimod leads to $\mathrm{G}_{2} / \mathrm{M}$ cell cycle arrest in TRAMP-C2 cells (A) TRAMP-C2 cells with or without imiquimod treatment were stained with PI and DNA contents were analyzed by flow cytometry. (B) The percentage of cells in each phase of the cell cycle is shown. (C) The protein level of cell cycle-related genes in the imiquimod-treated TRAMP-C2 cells was evaluated by western blot analysis.

imiquimod can induce direct apoptosis in various cancer cells $(13,17,18,20)$. To investigate whether imiquimod induces apoptosis in prostate cancer cells, TRAMP-C2 cells were treated with 10 or $20 \mu \mathrm{g} / \mathrm{ml}$ of imiquimod for $48 \mathrm{~h}$, stained 
A

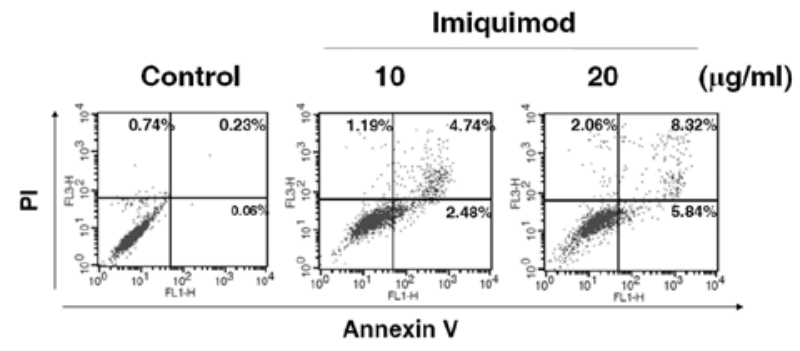

B

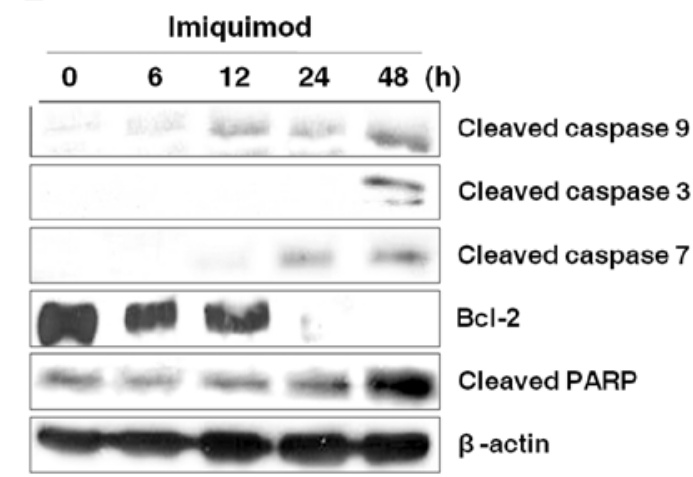

Figure 3. Imiquimod induces apoptosis in TRAMPC-2 cells. (A) TRAMP-C2 cells with or without imiquimod treatment were stained with PI and Annexin V-FITC and analyzed by flow cytometry. (B) The protein level of cell apoptosis-related genes in the imiquimod-treated TRAMP-C2 cells was evaluated by western blot analysis.
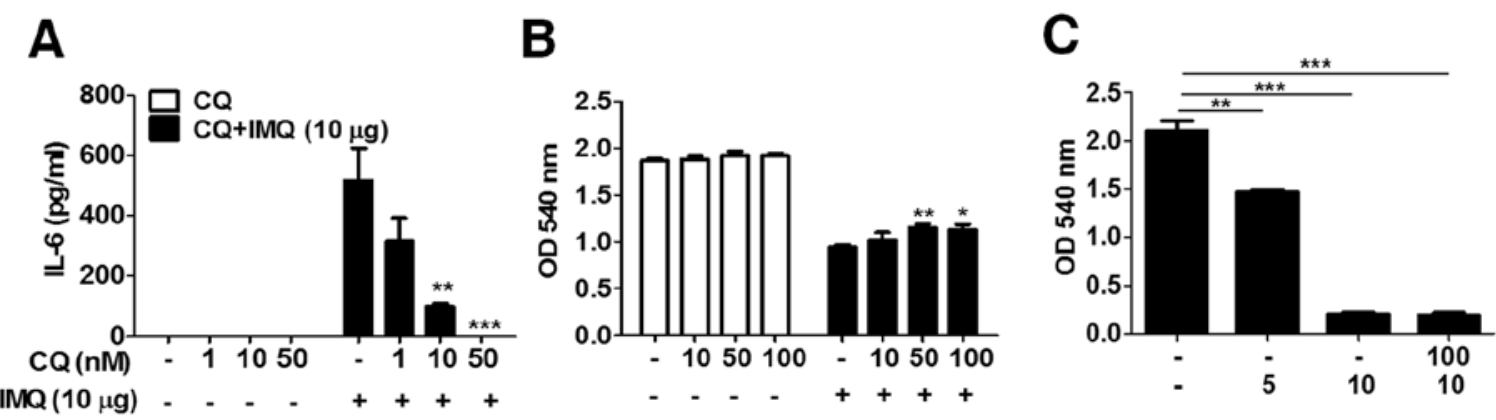

Figure 4. TLR7 is not required for the growth inhibition by imiquidmod in TRAMP-C2 cells. (A) Imiquimod-induced IL-6 production and (B and C) cell growth inhibition were evaluated with or without chloroquine pretreatment. (A) Culture supernatants from imiquimod-treated TRAMP-C2 cells were collected and IL-6 levels were measured by ELISA. Growth inhibition was measured (B) 3 days or (C) 9 days after imiquimod treatment by MTT assay (B). The results presented here are from one representative experiment of two independent experiments and are presented as mean $\pm \mathrm{SD}$. ${ }^{*} \mathrm{P}<0.05,{ }^{* *} \mathrm{P}<0.01,{ }^{* * * *} \mathrm{P}<0.001$.

with PI and Annexin V, and analyzed by flow cytometry. The percentage of Annexin V-positive cells was increased by the treatment of imiquimod dose-dependently (Fig. 3A). In addition, we examined the change of molecules associating with mitochondrial-dependent apoptosis pathway by western blot analysis. The anti-apoptotic molecule bcl-2 expression in TRAMP-C2 cells was reduced by imiquimod in a time-dependent manner (Fig. 3B). Imiquimod also led to cleavage of caspase-9, caspase- 3 and caspase- 7 by $48 \mathrm{~h}$ (Fig. 3B). Moreover, cleaved form of PARP was also increased by imiquimod in TRAMP-C2 cells (Fig. 3B). These findings indicate that imiquimod may lead to apoptosis in prostate cancer cells via the intrinsic pathway.

A TLR7 inhibitor chloroquine does not restore the growth inhibition by imiquimod in prostate cancer cells. Although imiquimod has been described as a TLR7 agonist, it is still unclear whether TLR7 is required for its antitumor effect. To clarify this, we performed an inhibitor assay using chloroquine, which is an anti-malarial drug to block activation of endosomal TLRs such as TLR3, 7 and 9 by inhibiting endosomal acidification (27). Our preliminary experiment showed that imiquimod enhanced the gene expression of
TLR7 and induced IL-6 production in TRAMP-C2 cells (data not shown). As shown in Fig. 4A, chloroquine inhibited imiquimod-induced production of IL-6 in the cells in a dosedependent manner. Although statistically significant, the restorative effect of chloroquine on imiquimod-induced inhibition of cell growth was minor (Fig. 4B). In an experiment with longer incubation time ( 9 days), chloroquine did not restore the growth inhibition by imiquimod in TRAMP-C2 cells (Fig. 4C).

Imiquimod inhibits in vivo growth of prostate cancer in mice. In vivo antitumor efficacy of imiquimod was evaluated in a mouse model s.c. implanted with TRAMP-C2 cells. On day 10 after tumor implantation, mice were daily treated with PBS or imiquimod $(50 \mu \mathrm{g})$ by intratumoral injection for 9 days. Tumor size was significantly reduced in imiquimod-treated mice, as compared with PBS-treated group (Fig.5A and B). Histologically, live tumor cells were compactly grown in PBS-treated mice (Fig. 5C). In contrast, in imiquimod-treated mice, necrotic area was broadly observed and cellular components were mostly dead cells (Fig. 5C). TUNEL staining revealed that apoptotic cells were increased by intratumoral injection of imiquimod in TRAMP-C2 cell-implanted mice (Fig. 5D). 
A

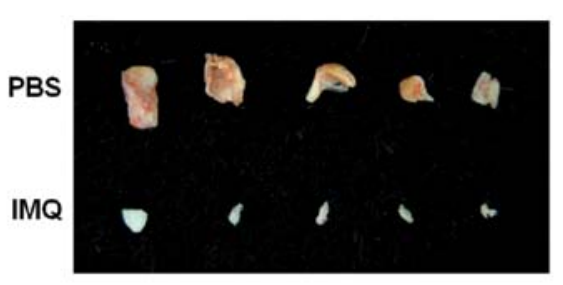

C

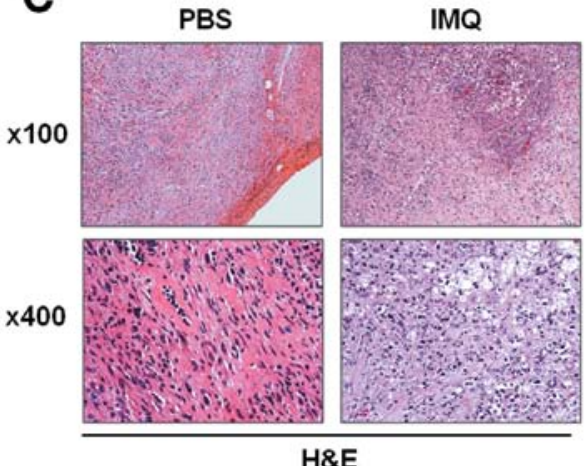

B

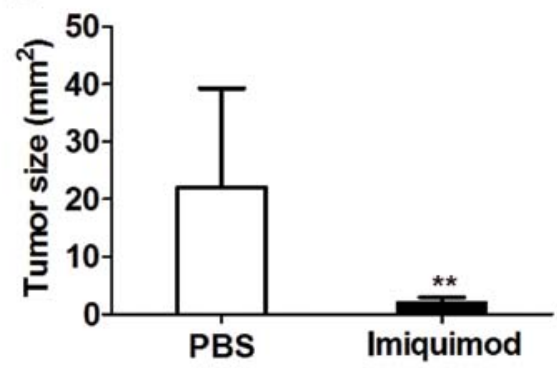

D

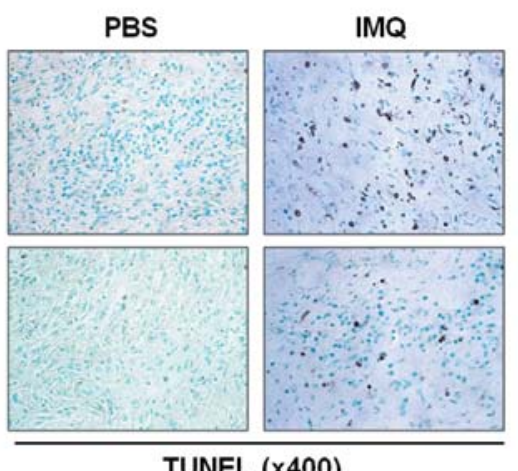

Figure 5. Imiquimod inhibits in vivo growth of prostate cancer in mice. (A) After cancer cell implantation, mice were intratumorally injected with PBS (upper) or imiquimod (lower). (B) The mean size of prostate tumor in each group is shown. Tumor mass section was evaluated histologically by (C) H\&E staining or (D) TUNEL staining. Statistical comparisons are made between the treated groups and the control groups injected with the same volume of the saline: ${ }^{* *} \mathrm{P}<0.01$.

\section{Discussion}

Although imiquimod is known as a TLR7 agonist, it can also induce cellular signaling TLR7-independently. Imiquimod can induce transcriptional activation of proinflammatory factors through adenosine receptor signaling (28). In addition, it activates p38, ERK and JNK MAPKs and induces apoptosis in primary keratinocytes independently of TLR7 and MyD88 (29). Schön et al suggest that imiquimod triggers inflammatory response via TLR7/8 or adenosine receptor signaling, whereas it induces direct apoptosis independently (28). In this study, an endosomal TLR inhibitor chloroquine inhibited imiquimod-induced production of IL- 6 in TRAMP-C 2 cells, whereas it did not affect cell growth inhibition by imiquimod. These results suggest that imiquimod may primarily induce growth inhibition in prostate cancer cells through a TLR7independent mechanism.

Regulation of cell cycle and apoptosis can be important targets for cancer chemotherapy (30). The anti-proliferative effects of imidazoquinolines family are mediated by cell cycle arrest and/or apoptosis in various cancer cells $(31,32)$. However, the pattern of cell cycle arrest caused by imidazoquinolines is different between the studies. An imidazoquinoline derivative NVP-BEZ235 inhibits the growth of T cell acute lymphoblastic leukemia in the $G_{0} / G_{1}$ phase of the cell cycle (33). In addition, imiquimod and resiquimod treatment of cancer cells decreased cell proliferation through $\mathrm{G}_{1} / \mathrm{S}$ phase arrest of the cell cycle through opioid growth factor receptor (OGFr) pathway (32). In contrast, treatment of EAPB0203, a member of the imidazo[1,2-a]quinoxalines, inhibited cell growth through
$\mathrm{G}_{2} / \mathrm{M}$ cell cycle arrest and apoptosis in HTLV-I-transformed and HTLV-I-negative malignant T cells and fresh ATL cells (31). In the present study, flow cytometry and western blot analysis revealed that imiquimod induces cell cycle arrest at $\mathrm{G}_{2} / \mathrm{M}$ phase in TRAMP-C2 cells. It remains to be clarified whether different pattern of imidazoquinolines-induced cell cycle arrest is due to difference of cancer cell types or structural difference of imidazoquinolines.

It is well known that imiquimod induces direct apoptosis in various cancer cells $(12,13,16,34)$. The mechanism of imiquimod-induced apoptosis in tumor cells seems to be mitochondria-dependent. In melanoma cells, the pro-apoptotic activity of imiquimod was independent of cell surface death receptors including CD95, TNF receptors or TRAIL (TNF-related apoptosis-inducing ligand) receptors $(12,13)$. Rather, it was depended on Bcl-2 degradation because melanoma cells overexpressing $\mathrm{Bcl}-2$ were relatively resistant against imiquimod-induced apoptosis as compared with their sham-transfected control cells (13). Apoptosis of tumor cells was abrogated by inhibition of caspase activation (13). Moreover, blocking the functions of membrane-bound death receptors did not affect the pro-apoptotic activity of imiquimod (12). Imiquimod resulted in release of mitochondrial cytochrome $c$ into the cytosol, which is a process that eventually leads to activation of caspase- 9 and caspase- 3 by proteolytic cleavage (35). In this study, imiquimod led to Bcl-2 degradation and cleavage of caspase-9, which are critical for intrinsic apoptosis. Therefore, as in other types of cancer, imiquimod seems to induce apoptosis in prostate cancer cells via mitochondria-dependent pathway. 
In this study, intratumoral injection of imiquimod effectively inhibited in vivo tumor growth in mice s.c. implanted with TRAMP-C2 cells. Although the number of apoptotic cells was increased in imiquimod-treated mice, in vivo antitumor action mechanism of imiquimod seems to be more complex. Several studies have demonstrated that cytokines such as type I IFN or cytotoxic T cells could participate in tumor destruction in vivo induced by imiquimod. Imiquimod can induce a profound tumordirected cellular immune response $(17,36)$. Sullivan et al revealed that antitumorigenic effect of imiquimod is mediated by upregulation of local IFN- $\alpha$ levels (16). Moreover, a recent in vivo study showed that imiquimod strongly enhances antigen-specific activation of antitumoral CD8 ${ }^{+}$ $\mathrm{T}$ cells (37). Collectively, it is likely that in vivo antitumor effect of imiquimod is achieved by direct apoptosis and enhancement of immune responses.

To our knowledge, this study is the first attempt to demonstrate that a direct treatment of imiquimod can significantly inhibit the growth of prostate cancer. Our current study revealed that imiquimod suppressed the proliferation of both mouse (TRAMP-C2) and human (PC3) prostate cancer cells in a TLR7-independent manner. Treatment of imiquimod resulted in $\mathrm{G}_{2} / \mathrm{M}$ phase cell cycle arrest and intrinsic apoptosis. Finally, we showed that imiquimod effectively inhibited tumor growth in mice s.c. implanted with TRAMP-C2 cells. These results suggest that imiquimod can be an effective therapeutic against locally generated prostate cancer.

\section{Acknowledgements}

This study was supported by a program (grant no. 2010-0002626) for Basic Research in Science and Engineering and by the World Class Institute (WCI) program (grant no. 2009-002) of the National Research Foundation of Korea (NRF) funded by the Ministry of Education, Science and Technology of Korea (MEST).

\section{References}

1. Jemal A, Murray T, Ward E, et al: Cancer statistics, 2005. CA Cancer J Clin 55: 10-30, 2005.

2. De Marzo AM, Platz EA, Sutcliffe S, et al: Inflammation in prostate carcinogenesis. Nat Rev Cancer 7: 256-269, 2007.

3. Pienta KJ and Smith DC: Advances in prostate cancer chemotherapy: a new era begins. CA Cancer J Clin 55: 300-318, 2005.

4. Maluf FC, Smaletz O and Herchenhorn D: Castration-resistant prostate cancer: systemic therapy in 2012. Clinics 67: 389-394, 2012.

5. Feldman BJ and Feldman D: The development of androgen-independent prostate cancer. Nat Rev Cancer 1: $34-45,2001$.

6. Rosenberg JE, Weinberg VK, Kelly WK, et al: Activity of second-line chemotherapy in docetaxel-refractory hormone-refractory prostate cancer patients. Cancer 110: 556-563, 2007.

7. Miller R, Gerster J, Owens M, Slade H and Tomai M: Review article imiquimod applied topically: a novel immune response modifier and new class of drug. Int J Immunopharmacol 21: $1-14,1999$.

8. Von Krogh G, Lacey C, Gross G, Barrasso R and Schneider A: European course on HPV associated pathology: guidelines for primary care physicians for the diagnosis and management of anogenital warts. Sex Transm Infect 76: 162-168, 2000.
9. Sidky YA, Borden EC, Weeks CE, Reiter MJ, Hatcher JF and Bryan GT: Inhibition of murine tumor growth by an interferon-inducing imidazoquinolinamine. Cancer Res 52: 3528-3533, 1992.

10. Hengge UR, Roth $S$ and Tannapfel A: Topical imiquimod to treat recurrent breast cancer. Breast Cancer Res Treat 94: 93-94, 2005.

11. Beutner KR, Geisse JK, Helman D, Fox TL, Ginkeld A and Owens ML: Therapeutic response of basal cell carcinoma to the immune response modifier imiquimod 5\% cream. J Am Acad Dermatol 41: 1002-1007, 1999.

12. Schön M, Bong AB, Drewniok C, et al: Tumor-selective induction of apoptosis and the small-molecule immune response modifier imiquimod. J Natl Cancer Inst 95: 11381149, 2003.

13. Schön MP, Wienrich BG, Drewniok C, et al: Death receptor-independent apoptosis in malignant melanoma induced by the small-molecule immune response modifier imiquimod. J Invest Dermatol 122: 1266-1276, 2004.

14. Berman B, Sullivan T, De Araujo T and Nadji M: Expression of Fas-receptor on basal cell carcinomas after treatment with imiquimod 5\% cream or vehicle. Br J Dermatol 149: 59-61, 2003.

15. Smith KJ, Germain M and Skelton H: Squamous cell carcinoma in situ (Bowen's disease) in renal transplant patients treated with 5\% imiquimod and 5\% 5-fluorouracil therapy. Dermatol Surg 27: 561-564, 2001.

16. Sullivan TP, Dearaujo T, Vincek V and Berman B: Evaluation of superficial basal cell carcinomas after treatment with imiquimod $5 \%$ cream or vehicle for apoptosis and lymphocyte phenotyping. Dermatol Surg 29: 1181-1186, 2003.

17. Smith EB, Schwartz M, Kawamoto H, et al: Antitumor effects of imidazoquinolines in urothelial cell carcinoma of the bladder. J Urol 177: 2347-2351, 2007.

18. Schwartz MJ, Liu H, Hwang DH, Kawamoto H and Scherr DS: Antitumor effects of an imidazoquinoline in renal cell carcinoma. Urology 73: 1156-1162, 2009.

19. Hayashi T, Crain B, Corr M, et al: Intravesical Toll-like receptor 7 agonist R-837: optimization of its formulation in an orthotopic mouse model of bladder cancer. Int J Urol 17: 483-490, 2010.

20. Asakura $M$ and Miura H: Imiquimod $5 \%$ cream for the treatment of nasal lesion of metastatic renal cell carcinoma. Dermatol Ther 24: 375-377, 2011.

21. Liu H, Schwartz MJ, Hwang DH and Scherr DS: Tumour growth inhibition by an imidazoquinoline is associated with c-Myc down-regulation in urothelial cell carcinoma. BJU Int 101: 894-901, 2008.

22. Kauffman EC, Liu H, Schwartz MJ and Scherr DS: Toll-like receptor 7 agonist therapy with imidazoquinoline enhances cancer cell death and increases lymphocytic infiltration and proinflammatory cytokine production in established tumors of a renal cell carcinoma mouse model. J Oncol 2012: 103298, 2012.

23. Foster BA, Gingrich JR, Kwon ED, Madias C and Greenberg NM: Characterization of prostatic epithelial cell lines derived from transgenic adenocarcinoma of the mouse prostate (TRAMP) model. Cancer Res 57: 3325, 1997.

24. Touny LH and Banerjee PP: Identification of both Myt 1 and Wee 1 as necessary mediators of the p21 independent inactivation of the cdc 2/cyclin B1 complex and growth inhibition of TRAMP cancer cells by genistein. Prostate 66: 1542-1555, 2006.

25. Toyoshima F, Moriguchi T, Wada A, Fukuda M and Nishida E: Nuclear export of cyclin B1 and its possible role in the DNA damage-induced G2 checkpoint. EMBO J 17: 2728-2735, 1998.

26. O'Connor PM: Mammalian G1 and G2 phase checkpoints. Cancer Surv 29: 151-182, 1997.

27. Sainathan SK, Bishnupuri KS, Aden K, et al: Toll-like receptor-7 ligand imiquimod induces type I interferon and antimicrobial peptides to ameliorate dextran sodium sulfate-induced acute colitis. Inflamm Bowel Dis 18: 955-967, 2012.

28. Schon MP, Schon M and Klotz KN: The small antitumoral immune response modifier imiquimod interacts with adenosine receptor signaling in a TLR7-and TLR8-independent fashion. J Invest Dermatol 126: 1338-1347, 2006.

29. Drobits B, Holcmann M, Amberg N, et al: Imiquimod clears tumors in mice independent of adaptive immunity by converting pDCs into tumor-killing effector cells. J Clin Invest 122: 575-585, 2012.

30. Hartwell LH and Kastan MB: Cell cycle control and cancer. Science 266: 1821-1828, 1994. 
31. Moarbess G, El-Hajj H, Kfoury Y, et al: EAPB0203, a member of the imidazoquinoxaline family, inhibits growth and induces caspase-dependent apoptosis in T-cell lymphomas and HTLV-I-associated adult T-cell leukemia/lymphoma. Blood 111: 3770-3777, 2008.

32. Zagon IS, Donahue RN, Rogosnitzky M and Mclaughlin PJ Imiquimod upregulates the opioid growth factor receptor to inhibit cell proliferation independent of immune function. Exp Biol Med (Maywood) 233: 968-979, 2008.

33. Chiarini F, Grimaldi C, Ricci F, et al: Activity of the novel dual phosphatidylinositol 3-kinase/mammalian target of rapamycin inhibitor NVP-BEZ235 against T-cell acute lymphoblastic leukemia. Cancer Res 70: 8097-8107, 2010.
34. Meyer T, Nindl I, Schmook T, Ulrich C, Sterry W and Stockfleth E: Induction of apoptosis by Toll-like receptor-7 agonist in tissue cultures. Br J Dermatol 149: 9-13, 2003.

35. Cryns V and Yuan J: Proteases to die for. Genes and development 12: 1551-1570, 1998.

36. Imbertson LM, Beaurline JM, Couture AM, et al: Cytokine induction in hairless mouse and rat skin after topical application of the immune response modifiers imiquimod and S-28463. J Invest Dermatol 110: 734-739, 1998.

37. Rechtsteiner G, Warger T, Osterloh P, Schild H and Radsak MP: Cutting edge: priming of CTL by transcutaneous peptide immunization with imiquimod. J Immunol 174: 2476-2480, 2005. 